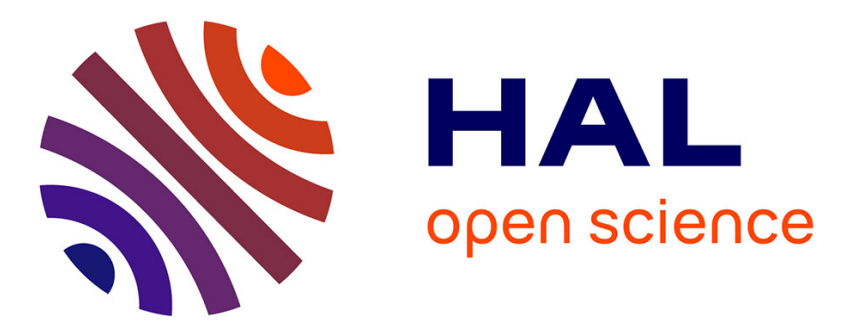

\title{
Magnetization reversal in exchange-coupled GdFe/TbFe studied by x-ray magnetic circular dichroism
}

\author{
S. Mangin, C. Bellouard, S. Andrieu, F. Montaigne, P. Ohresser, N. B
}

Brookes, B. Barbara

\section{- To cite this version:}

S. Mangin, C. Bellouard, S. Andrieu, F. Montaigne, P. Ohresser, et al.. Magnetization reversal in exchange-coupled GdFe/TbFe studied by x-ray magnetic circular dichroism. Physical Review B: Condensed Matter and Materials Physics (1998-2015), 2004, 70, pp.014401. 10.1103/PhysRevB.70.014401 . hal-02086069

\section{HAL Id: hal-02086069 \\ https://hal.univ-lorraine.fr/hal-02086069}

Submitted on 1 Apr 2019

HAL is a multi-disciplinary open access archive for the deposit and dissemination of scientific research documents, whether they are published or not. The documents may come from teaching and research institutions in France or abroad, or from public or private research centers.
L'archive ouverte pluridisciplinaire HAL, est destinée au dépôt et à la diffusion de documents scientifiques de niveau recherche, publiés ou non, émanant des établissements d'enseignement et de recherche français ou étrangers, des laboratoires publics ou privés. 


\title{
Magnetization reversal in exchange-coupled $\mathrm{GdFe} / \mathrm{TbFe}$ studied by $\mathrm{x}$-ray magnetic circular dichroism
}

\author{
S. Mangin, ${ }^{1}$ C. Bellouard,${ }^{1}$ S. Andrieu, ${ }^{1}$ F. Montaigne, ${ }^{1}$ P. Ohresser, ${ }^{2}$ N. B. Brookes,${ }^{2}$ and B. Barbara ${ }^{3}$ \\ ${ }^{1}$ Laboratoire de Physique des Matériaux, U.H.P-Nancy I, B.P 239 F-54506 Vandoeuvre Cedex, France \\ ${ }^{2}$ ESRF, B.P. 220 F-38043 Grenoble Cedex, France \\ ${ }^{3}$ Laboratoire de magnétisme Louis Néel, CNRS, B.P. 166, F-38042 Grenoble Cedex 9, France \\ (Received 25 July 2003; revised manuscript received 10 November 2003; published 2 July 2004)
}

\begin{abstract}
This work is dedicated to the study of magnetization reversal processes in a ferromagnetically exchange coupled ferrimagnetic/ferrimagnetic bilayer system made of a soft magnetic GdFe layer and a hard TbFe layer. Regular magnetization measurements and x-ray magnetic circular dichroism (XMCD) measurements performed at the ESRF on a $\operatorname{GdFe}(100 \mathrm{~nm}) / \mathrm{TbFe}(3 \mathrm{~nm})$ bilayer at different temperatures are presented and compared. The XMCD measurements allowed us to study separately the magnetic contribution of $\mathrm{Gd}, \mathrm{Tb}$, and $\mathrm{Fe}$ at the system interface. Evidence of the creation of an interface domain wall and its compression and pinning are given. In addition to quasistatic measurements, the interface magnetization reversal was also studied using XMCD aftereffect measurements. Both magnetization and XMCD measurements are explained by considering the formation of lateral domains during the interface magnetization reversal.
\end{abstract}

DOI: 10.1103/PhysRevB.70.014401

PACS number(s): 75.60.Ch, 75.60.Lr, 75.70.Rf, 78.20.Ls

\section{INTRODUCTION}

During the past ten years, a lot of work has been dedicated to the study of exchange-coupled (EC) bilayers. A variety of systems made of magnetic layers coupled by a positive or negative interface exchange interaction are concerned. The magnetic layers may be ferromagnetic, ferrimagnetic, or antiferromagnetic, magnetically soft or hard. The best known EC system is the antiferromagnetic/ferromagnetic (AFM/ FM) bilayer system that exhibits exchange bias phenomena after cooling through a blocking temperature $T_{b}$ under an applied field. ${ }^{1-3}$ One of the effects of exchange bias is to shift the hysteresis loop of the FM layers toward an exchange bias field $\left(H_{E}\right)$, which may be positive or negative. ${ }^{4}$ In addition exchange coupled materials also cover a variety of systems such as "spring magnets," ${ }^{5-9}$ "artificial antiferromagnets" 10 and others. 11,12 These systems have found technological applications in magneto-electronic devices. ${ }^{13,14}$

Despite the number of studies, the full understanding of the magnetization reversal processes taking place in EC systems is still debated as shown by the number of review papers on the subject. ${ }^{1-3}$ All studies clearly point out the major role played by the magnetic configuration at the interface. Some models are based on the presence of lateral domains in at least one of the layers, ${ }^{15}$ whereas other models suggest uniform lateral magnetization and the creation of a magnetic domain wall at the interface between the two layers. ${ }^{16-18}$ In magnetically hard and soft bilayers called "spring magnets," it is assumed that a magnetic domain wall is created at the interface as the magnetization of the soft layer reverses, leading to an antiparallel alignment of the two layers' magnetization. In this case uniform lateral magnetization is assumed. ${ }^{5-7,19}$ Whether this assumption is always valid or not has not been discussed. Moreover, very little work has been dedicated to the evolution of the interface magnetization as the hard-layer magnetization reverses. Consequently, crucial questions concerning EC systems still remain: what are the micromagnetic configurations adopted by the system (especially at the interface)? And how do those configurations develop with respect to the field, temperature, and time?

The aim of the present paper is to address these questions by studying the magnetic configuration at the interface of a $\mathrm{Gd}_{40} \mathrm{Fe}_{60} / \mathrm{Tb}_{55} \mathrm{Fe}_{45}$ "spring magnet" and its field, temperature, and time dependence. Magnetization reversal processes are investigated by combining conventional magnetization measurements and $\mathrm{x}$-ray magnetic circular dichroism (XMCD) measurements. This last technique is rather new for the investigation of magnetization reversal. ${ }^{20-26}$ The chemical selectivity of XMCD gives it obvious advantages over conventional methods which determine the averaged magnetic properties. Moreover, this surface-sensitive technique allows us to probe the bilayer interface only. Furthermore, we used XMCD to study dynamic effects by performing aftereffect measurements, which are to our knowledge the first of their kind. In Sec. II, the main characteristics of XMCD and superconducting quantum interference device (SQUID) techniques and a description of the measurement procedures are given. In Sec. III, we present experimental results performed to characterize the magnetic moments held by each species at the interface vicinity and compare the data to a theoretical calculation. Magnetization reversal processes are studied by comparing XMCD and SQUID results in Sec. IV. In this section, we deal successively with measurements performed at $100 \mathrm{~K}$ and $30 \mathrm{~K}$, first by using quasistatic measurements and second by focusing on aftereffect measurements. Finally in Sec. V, we draw some conclusions on the interface magnetic configurations and their temperature and field dependence.

\section{SAMPLE AND EXPERIMENTS}

The glass $/ \mathrm{Gd}_{40} \mathrm{Fe}_{60}(100 \mathrm{~nm}) / \mathrm{Tb}_{45} \mathrm{Fe}_{55}(3 \mathrm{~nm}) / \mathrm{Al}(3 \mathrm{~nm})$ sample was prepared by evaporation of the pure elements in 
a high-vacuum chamber. $\mathrm{Gd}_{40} \mathrm{Fe}_{60}$ and $\mathrm{Tb}_{55} \mathrm{Fe}_{45}$ are amorphous ferrimagnetic alloys obtained by coevaporation on a glass substrate kept at $77 \mathrm{~K}$ during the growth. The stoechiometry of the alloy was controlled by using a quartz microbalance to measure the evaporation rate. A $3 \mathrm{~nm} \mathrm{Al} \mathrm{layer}$ was used as a capping layer. The composition of the alloys was checked ex situ using an $\mathrm{x}$-ray analysis technique and they were found to be close to the nominal values. Amorphicity of the alloys was checked by transmission electron microscopy.

Magnetization measurements were achieved using a SQUID magnetometer that allows applied fields up to $70 \mathrm{kOe}$, in a temperature ranging from $400 \mathrm{~K}$ to $2 \mathrm{~K}$. The $\mathrm{x}$-ray absorption and XMCD measurements were carried out at the Dragon beam line ID12B at the ESRF, using the circular polarized light emitted by the helical undulator Helios I. The present experiments were performed on the Fe $L_{2,3}$ edges and the $\mathrm{Gd}$ and $\mathrm{Tb} M_{4,5}$ edges. The detection was performed by measuring the total drain current from the sample [total electron yield (TEY) (Ref. 27)]. This drain current is well known to be proportional to the absorption near the sample surface, since the escape depth of the ejected electrons is a few nanometers [around $2 \mathrm{~nm}$ for $\mathrm{Ni}, \mathrm{Co}$, and Fe (Ref. 28)]. Consequently, if the magnetization of the entire thin $\mathrm{TbFe}$ top layer is probed, only the very top part (2-3 $\mathrm{nm}$ ) of the GdFe underlayer at the $\mathrm{GdFe} / \mathrm{TbFe}$ interface contributes to XMCD signal.

From the convention usually adopted by the community, ${ }^{27}$ for a positive applied field (parallel to the wave vector of the x-ray beam), we call $I^{-}\left(I^{+}\right)$the absorption intensity measured for the right (left) circular polarization $\sigma^{-}\left(\sigma^{+}\right)$. Three kinds of absorption measurements were performed to probe the bilayer interface.

(i) Conventional XMCD spectra $\left(I^{-}-I^{+}\right)$are recorded while sweeping photon energy around a given element edge. They are obtained by flipping the x-ray polarization from right $\sigma^{-}$to left $\sigma^{+}$and keeping the field direction fixed. Indeed, although flipping the magnetic field with a fixed circular polarization is often used to get the XMCD signal, this is not appropriate to study irreversible magnetic reversal processes. The dichroic signals are corrected from the $30^{\circ}$ incidence angle (Fig. 1) and the incomplete polarization of the light. At the time the experiments were performed, the polarization rate was $85 \% \pm 5 \%$ at the $L_{2,3} \mathrm{Fe}$ edge, $92.5 \%$ $\pm 5 \%$ at the $M_{4,5} \mathrm{Gd}$ edge, and $94 \% \pm 5 \%$ at the $M_{4,5} \mathrm{~Tb}$ edge.

(ii) The XMCD signals at the Fe $L_{3}$ edge and at the Gd and $\mathrm{Tb} M_{5}$ edges are recorded as a function of the applied field. More precisely, we measured the intensity $\left(I^{-}-I^{+}\right)$ for two energies, one exactly at the edge and the other below the edge. The intensity difference between these two points is thus recorded versus field. The background contribution (which may also contain some dichroism signal coming from another edge, as in the case of $\mathrm{Gd}$ and $\mathrm{Tb}$ edges which are close in energy) is thus eliminated. Using this technique, we are able to record element-specific and thus compoundselective hysteresis loops. The presented hysteresis loops were obtained by plotting, as a function of the applied field,

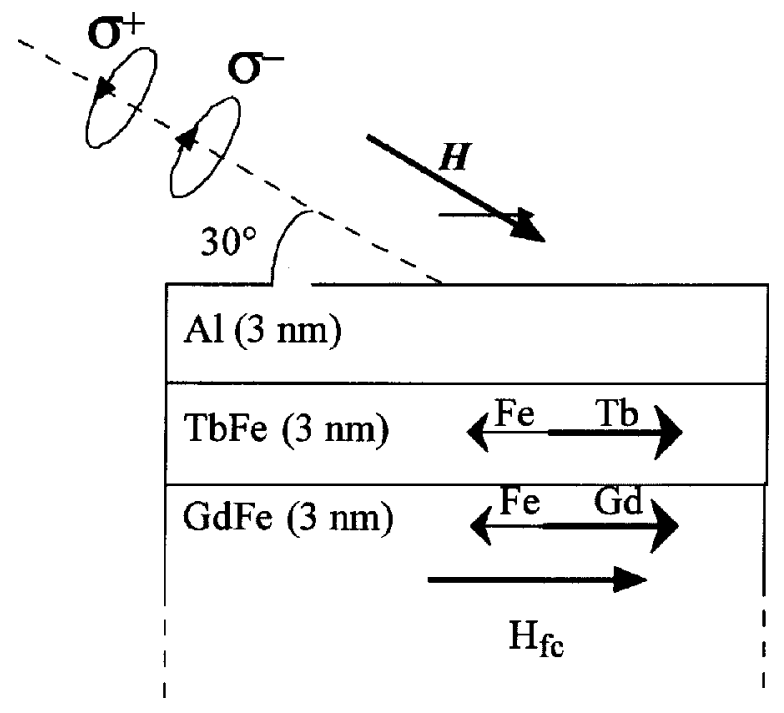

FIG. 1. X-ray magnetic circular dichroism experimental setup at the dragon beamline ID12B at the ESRF, using circular polarized $\sigma^{+}$and $\sigma^{-}$light. The sample is cooled down to the measurements temperature under a cooling field $\left(H_{f c}\right)$ applied along the $\mathrm{GdFe}$ easy axis in the film plane. During measurements, the incident beam is parallel to the applied magnetic field $(H)$ and makes a $30^{\circ}$ angle with the in plane cooling field axis. The sample is a $\mathrm{Gd}_{40} \mathrm{Fe}_{60}(100 \mathrm{~nm}) / \mathrm{Tb}_{55} \mathrm{Fe}_{45}(3 \mathrm{~nm}) / \mathrm{Al}(3 \mathrm{~nm})$ trilayer deposited on glass.

the XMCD signal normalized to the signal obtained at saturation $(\overline{\mathrm{XMCD}})$.

(iii) The XMCD signals at the $\mathrm{Fe} L_{3}$ edge and at the Gd and $\mathrm{Tb} M_{5}$ edges are recorded as a function of time $(t)$ for a fixed applied field. In a practical way, the sample is first saturated and the field is secondly swept to the measurement field. We are thus able to record element-specific interface magnetization reversal in real time and to perform aftereffect measurements.

Also it should be noted that, if the spin to orbital magnetic moment ratio does not change during the magnetization reversal process (and this is a reasonable assumption), the variation of $I^{+}$(or $I^{-}$) with field or time is strictly proportional to the variation of the magnetic moment projected along the light direction.

In summary, $\mathrm{XMCD}$ is an element-specific technique to study the $\mathrm{GdFe} / \mathrm{TbFe}$ interface magnetization which may be compared to SQUID measurements for which the projection along the field axis of the whole bilayer magnetization is averaged. The surface areas probed by the two kinds of experiments are of the same order of magnitude-i.e., approximately a few square millimeters. For the XMCD signal measurements, the integration time needed for a measurement $(0.1-0.5 \mathrm{~s})$ is at least one order of magnitude quicker than the time between two consecutive measurements. Thus, a few seconds separate two measurements when XMCD signals versus fields are measured, whereas it takes about a minute using SQUID.

In the XMCD experimental setup, the magnetic field is applied parallel to the direction of the incident $\mathrm{x}$-ray beam. Consequently, the best geometry to perform the magnetic 
characterization should be with the applied field and the incident X-ray beam lying in the sample plane. However, for the TEY detection mode, some saturation effects occur for low angle $(\alpha)$ between the incident beam and the surface. ${ }^{28}$ The best compromise was then to set $\alpha$ to $30^{\circ}$. In the following, the mentioned magnetic field values $H$ are the values of the field projection on the film plane $[H \cos (\alpha)]$. All XMCD experiments are treated as if only the in-plane component is relevant and that the perpendicular component can be neglected. This is indeed a fair approximation since $H \sin \alpha$ $<0.5 \mathrm{kOe} \ll 4 \pi M s \approx 15 \mathrm{kOe}$.

\section{SAMPLE AND INTERFACE MAGNETIC CHARACTERISTICS}

In $\mathrm{Gd}_{40} \mathrm{Fe}_{60}$ and $\mathrm{Tb}_{55} \mathrm{Fe}_{45}$, the coupling between $\mathrm{Fe}$ and the rare-earth $(\mathrm{Gd}$ and $\mathrm{Tb})$ spins is antiferromagnetic and for these two compositions, the contribution of the rare-earth moments to magnetization is dominant. ${ }^{29}$ In the GdFe soft magnetic layer, a uniaxial anisotropy axis in the film plane is induced by specific growth conditions. ${ }^{30}$ However, TbFe is a hard amorphous sperimagnetic alloy whose net magnetization is fixed at low temperature along the field cooling direction. Because the exchange coupling between the layers is dominated by $\mathrm{Fe}-\mathrm{Fe}$ ferromagnetic interactions, the coupling between the net magnetization of the two layers is ferromagnetic. $\mathrm{Gd}_{40} \mathrm{Fe}_{60} / \mathrm{Tb}_{55} \mathrm{Fe}_{45}$ is then a "spring magnet" system in which the $\mathrm{TbFe}$ layer is considered as the pinning layer.

XMCD experiments were first performed to get information on the average direction and amplitude of the interface atomic magnetic moments of each species at $100 \mathrm{~K}$. XMCD spectra at $\mathrm{Fe}, \mathrm{Tb}$, and $\mathrm{Gd}$ edges are presented in Fig. 2. From these measurements, we immediately see that the moments on the rare earths are antiparallel to the moment on the iron, since the XMCD Fe spectrum is reversed compared to the $\mathrm{Gd}$ and $\mathrm{Tb}$ ones. Moreover, the $L_{2,3} \mathrm{Fe}$ edge shape is typical of a metallic final band, which means that Fe and, consequently, the GdFe and TbFe layers are not oxidized. To obtain the average magnetic moments for each chemical species, we apply the sum rules ${ }^{31}$ using the spectra analysis proposed in Ref. 32. The integrated absorption and XMCD curves needed for calculating the orbital and spin moments ${ }^{32}$ are also shown in Fig. 2. The moments per holes are finally calculated including the circular polarization rate and the $30^{\circ}$ angle between the in-plane magnetization and the light direction. In the case of $\mathrm{Fe}$, the number of holes in the $3 d$ final bands of $\mathrm{Fe}$ in both $\mathrm{GdFe}$ and $\mathrm{TbFe}$ alloys is not known. We consequently chose the number of holes obtain in bulk bcc Fe [3.4 holes/atom (Ref. 32)]. We neglected the $\left\langle T_{z}\right\rangle$ contribution as usually assumed in $3 d$ metals. ${ }^{33}$ We then obtained an average value of spin $\left(m_{s}\right)$, orbital $\left(m_{l}\right)$, and total $\left(m_{\text {tot }}\right)$ magnetic moments of $\mathrm{Fe}$ over the TbFe and GdFe layers as

$$
\begin{gathered}
\text { Fe }: m_{s}=1.13 \pm 0.04, \quad m_{l}=0.018 \pm 0.01, \\
m_{\mathrm{tot}}=m_{s}+m_{l}=1.15 \pm 0.05 \mu_{B} / \mathrm{at} .
\end{gathered}
$$

Note that the weighted average $\mathrm{Fe}$ magnetic moments obtained mainly arise from the TbFe layer. In the case of the $M_{4,5} \mathrm{Gd}$ and $\mathrm{Tb}$ transitions, the number of holes is exactly

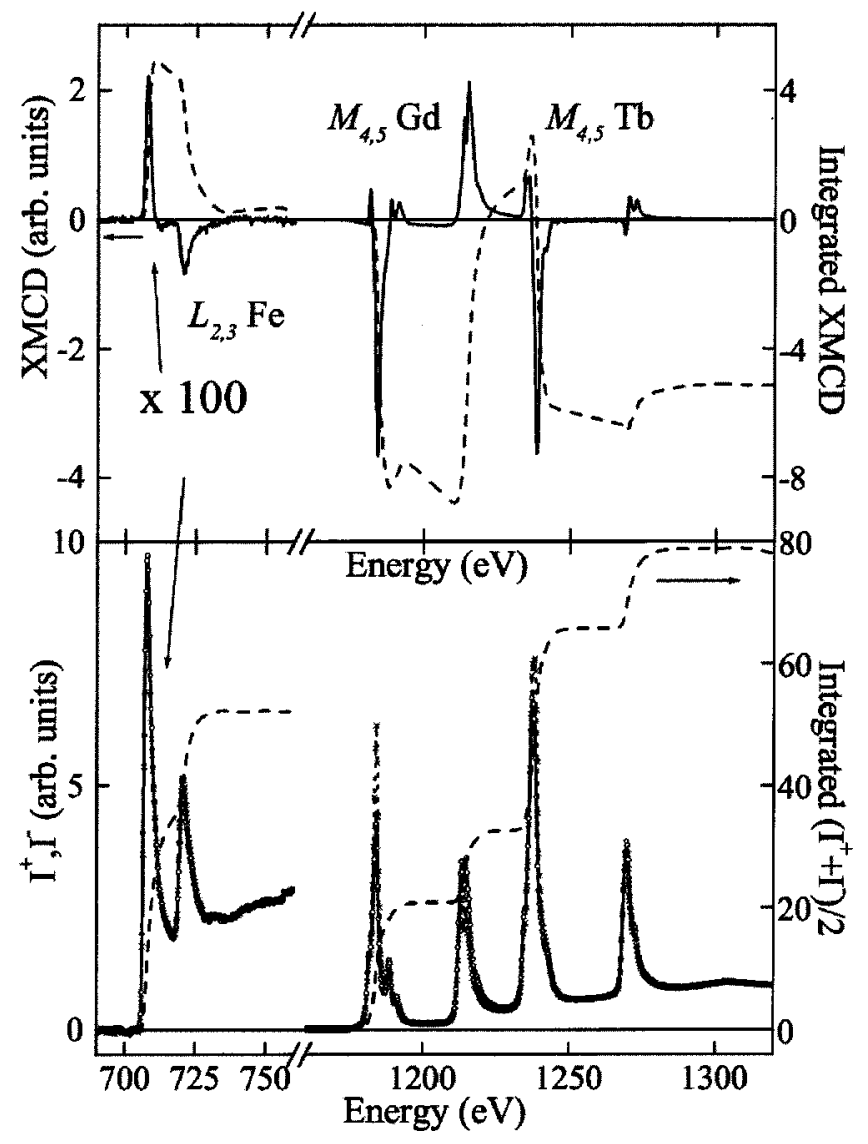

FIG. 2. (a) XMCD signals (solid lines) at the Fe $L_{2,3}$, Gd $M_{4,5}$ and $\mathrm{Tb} M_{4,5}$ edges obtained at $100 \mathrm{~K}$ for both circular polarizations $\sigma+$ and $\sigma^{-}$and integrated XMCD signal (dashed line) needed for the sum rules application (Ref. 32). (b) Absorption intensities $I^{+}$ (circle) and $I^{-}$(cross) at the Fe $L_{2,3}, \mathrm{Gd} M_{4,5}$ and $\mathrm{Tb} M_{4,5}$ and corresponding integrated $\left(I^{+}+I^{-}\right) / 2$ intensity needed for the sum rules application (Ref. 32).

known since the final state is the $4 f$ localized state ( $n_{\text {holes }}=7$ for $\mathrm{Gd}$ and 6 for $\mathrm{Tb}$ ). In the case of $\mathrm{Gd}$, the $\left\langle T_{z}\right\rangle$ contribution is exactly zero since $L=0$. In the case of $\mathrm{Tb}$, the $\left\langle T_{z}\right\rangle$ contribution must not be neglected since $\left\langle T_{z}\right\rangle /\left\langle S_{z}\right\rangle=-1 / 9$ which leads to a correction factor $\left(1+3\left\langle T_{z}\right\rangle /\left\langle S_{z}\right\rangle\right)^{-1}=3 / 2$ (Ref. 31). We finally obtained

$$
\begin{gathered}
\mathrm{Gd}: \quad m_{s}=6.5 \pm 0.2, \quad m_{l}=-0.15 \pm 0.3, \\
m_{\mathrm{tot}}=6.2 \pm 0.5 \mu_{B} / \text { at }, \\
\mathrm{Tb:} \quad m_{s}=2.3 \pm 0.3, \quad m_{l}=1.2 \pm 0.2, \\
m_{\mathrm{tot}}=3.5 \pm 0.5 \mu_{B} / \text { at. }
\end{gathered}
$$

The error bars on the magnetic moment values on $\mathrm{Gd}$ and $\mathrm{Tb}$ are quite large due to the proximity of both edges. Indeed, one may clearly observe in Fig. 2 that the Tb edge starts at an energy for which there is still some remaining dichroic signal at the tail of the $M_{4} \mathrm{Gd}$ edge. However, the accuracy is sufficient to compare these results with expected magnetic moments. 


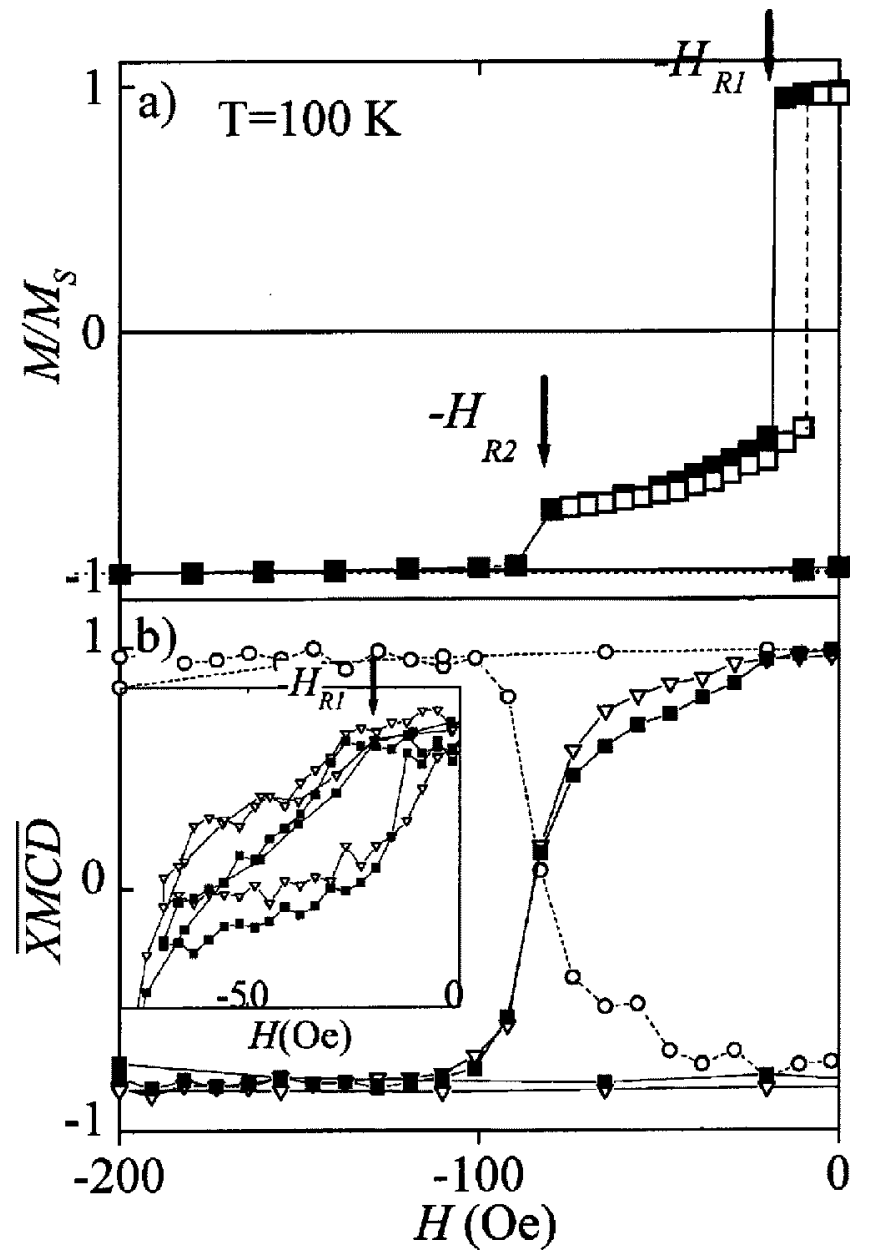

FIG. 3. Magnetic measurements performed at $100 \mathrm{~K}$ on Glass $/ \mathrm{Gd}_{40} \mathrm{Fe}_{60}(100 \mathrm{~nm}) / \mathrm{Tb}_{55} \mathrm{Fe}_{45}(3 \mathrm{~nm}) / \mathrm{Al}(3 \mathrm{~nm})$ after saturating the sample at $300 \mathrm{~K}$ under $1 \mathrm{kOe}$ applied along the GdFe easy axis and then cooling the sample under $H_{f c}=1 \mathrm{kOe}$ along the same direction. (a) Normalized SQUID magnetization $\left(M / M_{S}\right)$ hysteresis loop. Solid square $(\boldsymbol{\square})$ : major loop performed by sweeping the field from $1 \mathrm{kOe}$ to $-1 \mathrm{kOe}$ and back to $1 \mathrm{kOe}$; Open square $(\square)$ : minor loop obtained by sweeping the field from $1 \mathrm{kOe}$ to -75 Oe to 1 kOe. (b) Normalized XMCD signals ( $\overline{\mathrm{XMCD}}$ ) at the $\mathrm{Fe} L_{2,3}$ edges (open circle) and the Gd, (solid square) and Tb (open triangle) $M_{4,5}$ edges collected as a function of the applied field. The applied field was swept from $1 \mathrm{kOe}$ to -500 Oe back to $1 \mathrm{kOe}$. In inset, minor loop for a field swept from $1 \mathrm{kOe}$ to -75 Oe back to $1 \mathrm{kOe}$.

In theory, the atomic $4 f$ magnetic moments on $\mathrm{Gd}$ are $m_{s}=7 \mu_{B} /$ at and $m_{l}=0(J=S=7 / 2$ and $L=0)$ and on $\mathrm{Tb}$, $m_{s}=6 \mu_{B} /$ at and $m_{l}=m_{s} / 2=3 \mu_{B} /$ at $(J=6$ and $S=L=3)$. However, due to the sperimagnetic structure of the $\mathrm{TbFe}$ alloy, the $\mathrm{Tb}$ average magnetic moment is reduced by a factor $2 .^{34,35} \mathrm{~A} \mathrm{~Tb}$ magnetic moment close to $4.5 \mu_{B} /$ at is thus expected. From XMCD measurements a close-to-zero orbital magnetic moment in Gd and a spin moment which is twice the orbital moment in $\mathrm{Tb}$ are obtained, which is in total agreement with theory. Moreover, a very good agreement is obtained on the average total magnetic moments values of both Gd and Tb considering that the theoretical values overestimate the experimental results, since (i) the measurements were carried out at $100 \mathrm{~K}$ and not $0 \mathrm{~K}$, (ii) the two alloys are

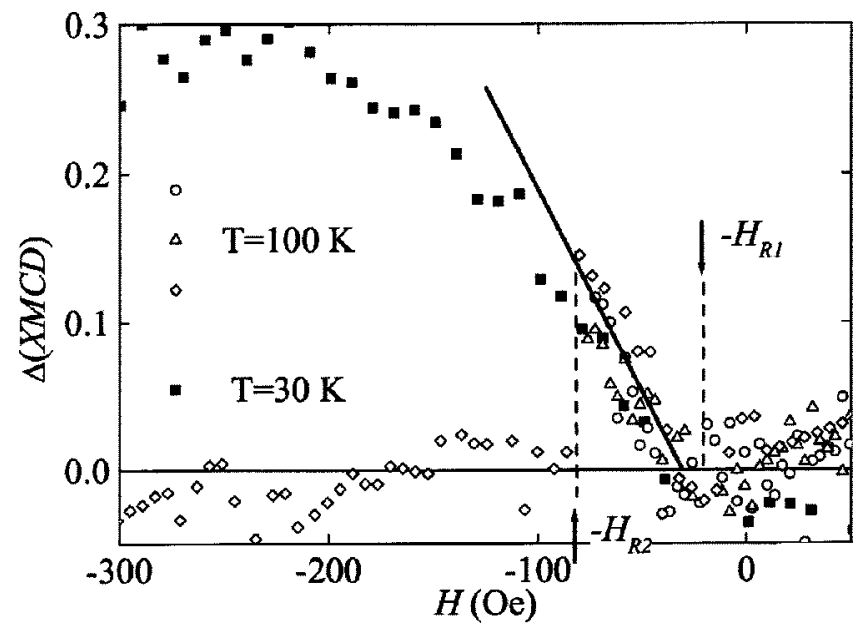

FIG. 4. The difference between the two normalized XMCD signal ( $\overline{\mathrm{XMCD}}$ ) measured at the $M_{4,5}$ edge of Gd and Tb: $\Delta(\mathrm{XMCD})$ is plotted as a function of the applied field $H . \Delta(\mathrm{XMCD})$ values deduced from a major loop (open diamond) and from a minor loop while sweeping the field from $1 \mathrm{kOe}$ to $-75 \mathrm{Oe}$ (open circle) and sweeping the field back from -75 to $1 \mathrm{kOe}$ (open triangle) obtained at $100 \mathrm{~K}$, are presented. $\Delta(\mathrm{XMCD})$ results from $30 \mathrm{~K}$ measurements (solid square) are compared to the one obtained at $100 \mathrm{~K}$.

amorphous $\mathrm{Gd}$ and $\mathrm{Tb}$, and (iii) XMCD saturation effects which can be estimated between $10 \%$ and $20 \%$ (Ref. 28) are not taken into account. In the case of Fe the magnetic moments values are coherent with the one obtained by Hansen et $a .^{29}$ Also those values are consistent with the alloys magnetization deduced from SQUID measurements for a 100-nm $\mathrm{Gd}_{40} \mathrm{Fe}_{60}$ layer and a $100-\mathrm{nm} \mathrm{Tb}_{55} \mathrm{Fe}_{45}$ layer. We may then conclude that no modification of the magnetic moments held by the different chemical species is observed in the vicinity of the interface.

\section{MAGNETIZATION REVERSAL RESULTS AND DISCUSSION}

\section{A. Quasistatic measurements}

Magnetization reversals occurring at $100 \mathrm{~K}$ are first studied. The results obtained by combining SQUID and XMCD experiments are shown in Fig. 3. For both techniques the same experimental procedure was used: The sample was saturated at $300 \mathrm{~K}$ under $1 \mathrm{kOe}$ applied field along the GdFe easy axis and was cooled down to the measurement temperature under a cooling field $H_{f c}=1 \mathrm{kOe}$ along the same direction. The field was then swept along the GdFe easy axis for SQUID and $30^{\circ}$ off the plane for XMCD (see Sec. II and Fig. 1). SQUID measurements reveal that the sample is saturated until a negative field $-H_{R 1}$. This saturation at the interface [as sketched in Fig. 7(a)] is confirmed by XMCD measurements, since the normalized signals (XMCD) of the rare earths are equal to +1 and the one of iron is equal to -1 .

For $H=-H_{R 1}$, an abrupt magnetization drop corresponding to about $80 \%$ of the sample magnetization is observed in the SQUID measurement without apparent incidence on the XMCD signals. This difference confirms that 


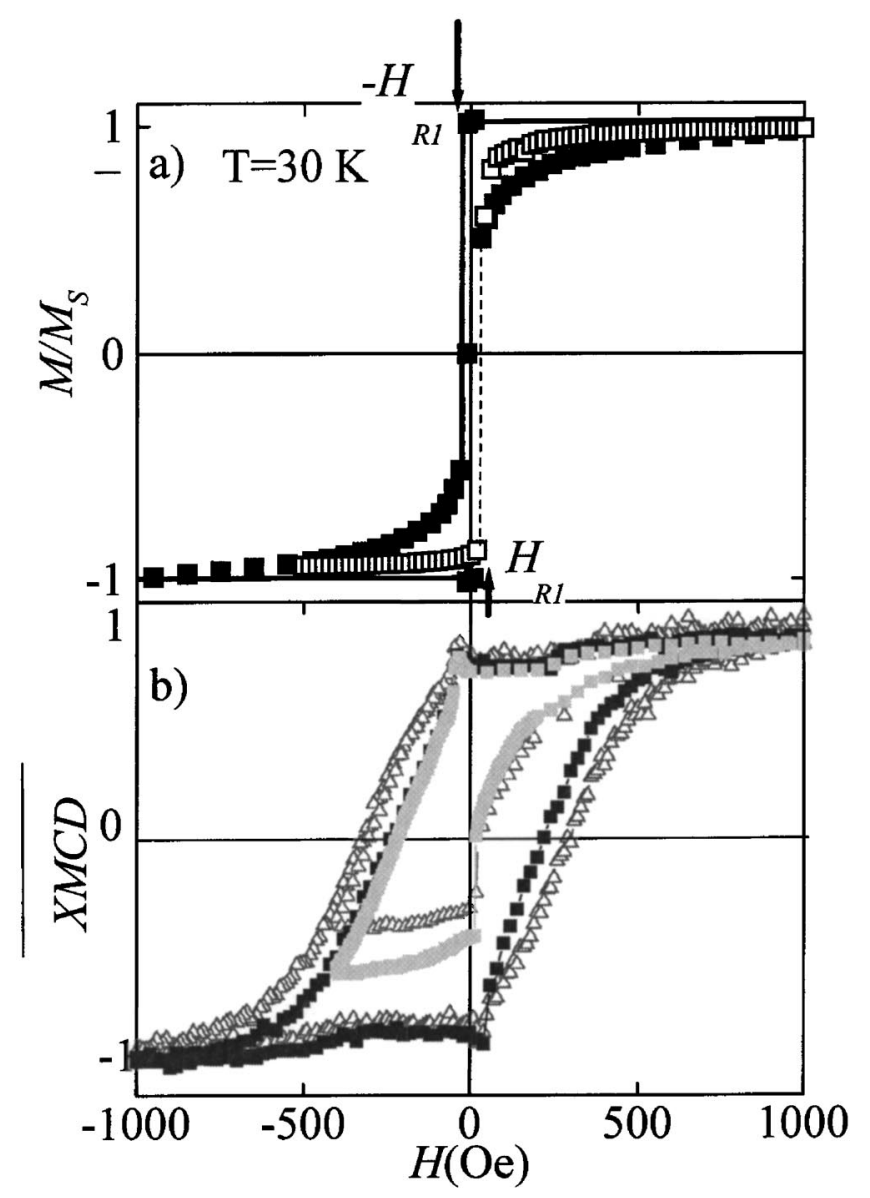

FIG. 5. Magnetic measurements performed at $30 \mathrm{~K}$ on Glass $/ \mathrm{Gd}_{40} \mathrm{Fe}_{60}(100 \mathrm{~nm}) / \mathrm{Tb}_{55} \mathrm{Fe}_{45}(3 \mathrm{~nm}) / \mathrm{Al}(3 \mathrm{~nm})$ after saturating the sample at $300 \mathrm{~K}$ under $1 \mathrm{kOe}$ applied along the GdFe easy axis and then cooling the sample under $H_{f c}=1 \mathrm{kOe}$. (a) Normalized SQUID magnetization $\left(M / M_{S}\right)$ hysteresis loop: Major loop [solid square $(\boldsymbol{\square})$ ], performed by sweeping the field from $1 \mathrm{kOe}$ to -1 kOe and back to $1 \mathrm{kOe}$. Minor loop [open square ( $\square)$ ] obtained by sweeping the field from $1 \mathrm{kOe}$ to -500 Oe to $1 \mathrm{kOe}$. (b) Normalized XMCD signals ( $\overline{\mathrm{XMCD}}$ ) at the Gd (solid square) and Tb (open triangle) $M_{4,5}$ edges collected as a function of the applied field at $100 \mathrm{~K}$. In the major loop the applied field is swept from $1 \mathrm{kOe}$ to $-1 \mathrm{kOe}$ to $1 \mathrm{kOe}$ and in the minor loop from $1 \mathrm{kOe}$ to -400 Oe to 1 kOe.-

the part of the sample probed is totally different in the two experiments. SQUID measurements point out that the major part of the GdFe layer magnetization reverses toward the field, whereas XMCD measurements indicate that GdFe magnetization at the interface is kept parallel to the $\mathrm{TbFe}$ magnetization, both still pointing along the cooling field direction. This implies the formation of a $180^{\circ}$ interface domain wall (DW) in the thickness of the GdFe layer which tends to minimize the exchange interaction [sketched in Fig. 7(b)]. The presence of this type of Bloch wall with a propagation vector perpendicular to the film plane has been experimentally demonstrated for similar bilayers with a thicker $\mathrm{TbFe}$ layer by polarized neutron reflectometry ${ }^{36}$ and magnetoresistance measurements. ${ }^{19}$ Their presence is also well established in similar exchange spring bilayers. ${ }^{5-7}$ The DW is then a Bloch wall. Given that GdFe has a much lower anisotropy constant than TbFe, it is consistent that the DW sits mainly inside the GdFe layer. The DW thickness expected in $\mathrm{GdFe}$ is around $50 \mathrm{~nm}$ (Ref. 7); the magnetization in the last nanometers of the GdFe layer probed by XMCD is then forming one end of the DW (magnetization pointing along the positive field direction).

For $-H_{R 1}>H>-H_{R 2}$ the smooth decrease of the magnetization observed by SQUID and the decrease of the XMCD signal from Gd are in agreement with a DW "compression" effect. This effect is due to the fact that as the field amplitude is increased, the DW size $(\delta)$ decreases, leading to the decrease of the total magnetization observed in SQUID measurements. Consequently, as the DW is compressed, each moment forming the DW rotates closer to the field direction. That explains the decrease of the signal coming from $\mathrm{Gd}$.

Another important feature given by XMCD measurement is that in this field range the TbFe magnetization also decreases and that the normalized GdFe magnetization decreases faster than the $\mathrm{TbFe}$ one. This effect may be explained in terms of interface DW if we consider that the DW at the interface is not only compressed but also penetrates in the TbFe layer [Figs. 7(b) and 7(c)]. Indeed, if the DW enters the $\mathrm{TbFe}$ layer, Gd magnetic moments should point closer to the field direction than the Tb one. While the DW is compressed the angle between two consecutive spins increases. Consequently, the average angle between the $\mathrm{Tb}$ moments inside the 3-nm TbFe layer and the $\mathrm{Gd}$ moments at the interface probed by XMCD should increase. To quantify this effect, we propose to look at the difference $\Delta(\mathrm{XMCD})$ between the two normalized XMCD signals $\overline{\mathrm{XMCD}}$ from $\mathrm{Tb}$ and $\mathrm{Gd}$ versus $H$. The $\Delta(\mathrm{XMCD})$ obtained from the experiments performed at 100 and $30 \mathrm{~K}$ are plotted in Fig. 4. $\Delta(\mathrm{XMCD})$ is expected to be equal to zero if the $\mathrm{Tb}$ and $\mathrm{Gd}$ moments at the interface are parallel and increase as the angle between the two types of moments at the interface increases. Also $\Delta(\mathrm{XMCD})$ should be equal to zero if no DW enters the $\mathrm{GdFe} / \mathrm{TbFe}$ interface and increase as an interface DW enters the TbFe layer and compress. Figure 4 shows that this is actually the case. Moreover, $\Delta(\mathrm{XMCD})$ is found to increase linearly with field during the compression stage.

The jump observed at $-H_{R 2}$ in both XMCD and SQUID measurements is associated with the magnetization reversal of the $\mathrm{GdFe} / \mathrm{TbFe}$ interface, which annihilates the interface DW. For $H=-H_{R 2}$, both rare-earth signals drop simultaneously. After the jump, the sample is saturated in the field direction [Fig. $7(\mathrm{~g})]$, the signals coming from the rare earths are minimum, and the signal coming from iron maximum and $\triangle(\mathrm{XMCD})$ is equal to zero. This magnetization reversal was studied in more detail using XMCD aftereffect measurements.

The minor loops performed with both techniques [Fig. 3(a) and inset of Fig. 3(b)] show that sweeping the field back for $H>-H_{R 2}$ (before the second magnetization reversal) permits us to reverse the GdFe layer magnetization towards the positive field direction in a negative field. A negative exchange bias field is then observed. The sign of the exchange bias field $H_{E}$ is consistent with the positive interface exchange coupling and its amplitude in agreement with the presence of an interface DW (Ref. 7) as proposed by Mauri et al.: ${ }^{16}$ 


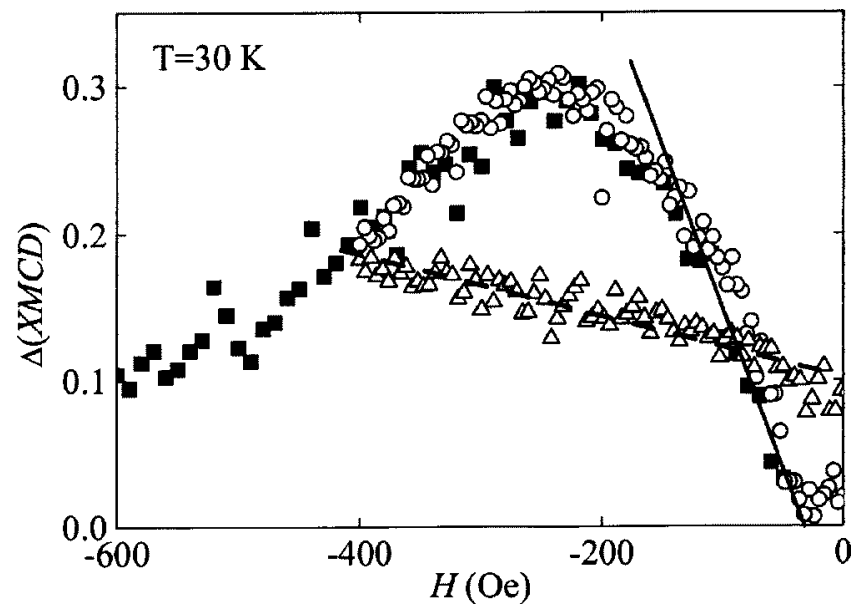

FIG. 6. Field dependences of the $\Delta(\mathrm{XMCD})$ values deduced from a major loop (solid square) and from the minor loop while sweeping the field from $1 \mathrm{kOe}$ to -400 Oe (open circle) and back from -400 Oe to $1 \mathrm{kOe}$ (open triangle) obtained at $30 \mathrm{~K}$.-

$$
\left|H_{E}\right| \approx \frac{\sigma}{2 M_{\mathrm{GdFe}} t_{\mathrm{GdFe}}} \approx 25 \mathrm{Oe}
$$

with $\sigma=0.7 \pm 0.3 \mathrm{erg} / \mathrm{cm}^{2}$ : the domain wall surface energy inside the $t_{\mathrm{GdFe}}=100 \pm 10 \mathrm{~nm}$ thick GdFe layer and $M_{\mathrm{GdFe}}$ $=1000 \pm 100 \mathrm{emu} / \mathrm{cm}^{3}$ its magnetization. XMCD and SQUID results show that the XMCD signal from Gd and the total magnetization slowly increase while the field is swept back. This can be understood in term of DW "decompression." Indeed, during this stage $\Delta$ (XMCD) decreases following a similar field dependence that observed during "compression."

SQUID and more clearly XMCD measurements shows that the "compression-decompression" process is not reversible. During the DW decompression, the variation of Gd and $\mathrm{Tb}$ XMCD signals is much weaker than during compression. As a consequence, at this stage, an opening of the $\overline{\mathrm{XMCD}}$ hysteresis loops is observed [inset of Fig. 3(b)]. This tends to prove that some of the $\mathrm{GdFe} / \mathrm{TbFe}$ interface magnetization is pinned. Since $\mathrm{TbFe}$ is a random anisotropic hard magnetic material, one may expect a pinning of the TbFe magnetization leading to the pinning of the DW. Following the DW decompression, the GdFe layer magnetization is reversed (before zero field is reached) and both rare-earth XMCD signals reach their maximum. The sample is then saturated in the positive field direction.

In summary, the $100 \mathrm{~K}$ measurements give evidence for (i) the creation of an interface DW as GdFe layer reverses for $H=-H_{R 1}$, (ii) the compression and the decompression of this DW which penetrates the TbFe layer for $-H_{R 1}>H>$ $-H_{R 2}$, (iii) the pinning of the $\mathrm{DW}$ at $\mathrm{TbFe} / \mathrm{GdFe}$ interface vicinity, and (iv) the reversal of the $\mathrm{TbFe} / \mathrm{GdFe}$ interface magnetization leading to the annihilation of the DW for $H=-H_{R 2}$. Note that this magnetization behavior may be understand by considering only a uniform lateral magnetization in the bilayer. a)

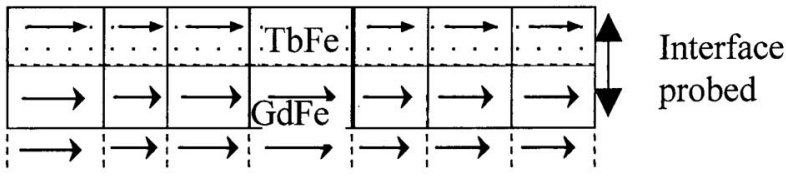

b)

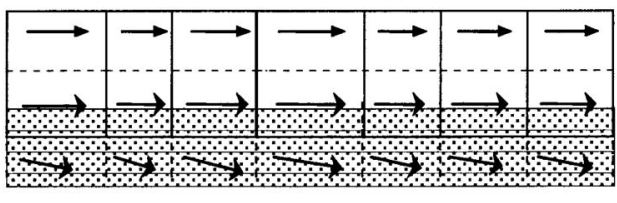

c)

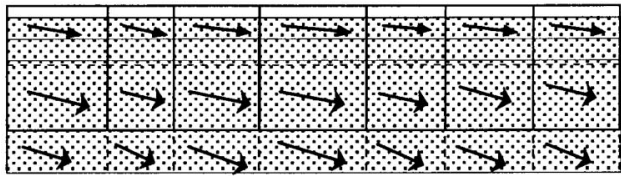

d)

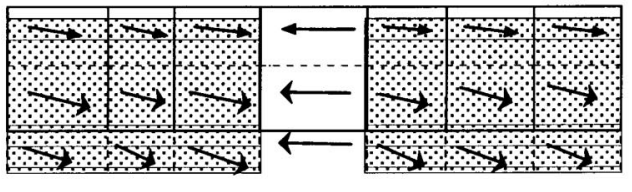

e)

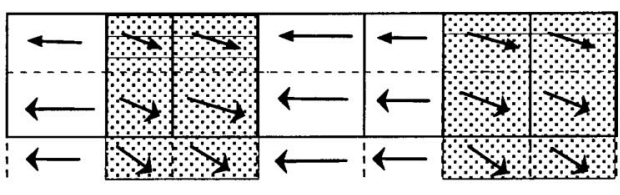

f)
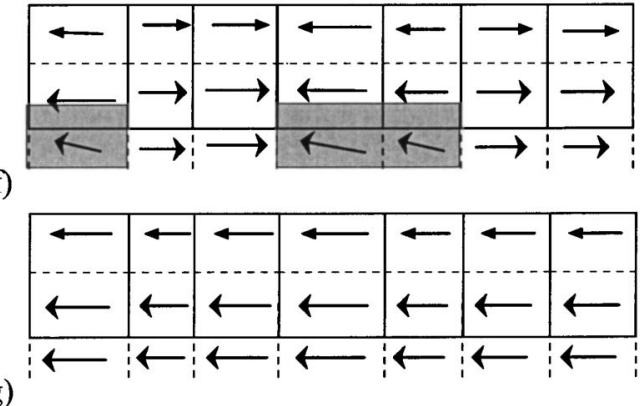

FIG. 7. Schematic magnetic configurations at the GdFe/TbFe interface. The position of the interface domain wall is colored in gray: (a) $H>-H_{R 1}$ : The sample is saturated along the cooling field direction (positive field direction). (b) $H=-H_{R 1}$ : a DW is present in the $\mathrm{GdFe}$ layer and is stopped at the $\mathrm{GdFe} / \mathrm{TbFe}$ interface (all domains are in the DW state). (c) $H<-H_{R 1}$ : The DW is compressed and penetrates in the TbFe layer. (d) One domain switches from a compressed DW state to a saturated state ( $\mathrm{Sat}^{-}$state). (e) A few domains are in saturated state and the other are in a compressed DW state. (f) GdFe magnetization reversal switches all domains in (e) from one state to the other. $(\mathrm{g})$ The sample is saturated in the negative field direction.

Measurements at $30 \mathrm{~K}$ using both XMCD and SQUID techniques are shown in Fig. 5. In the low-field regime $(H$ $>-100 \mathrm{Oe}) \mathrm{XMCD}$ and SQUID measurements confirm the creation, the compression, and the pinning of a DW as observed for $100 \mathrm{~K}$. Indeed, the sample is kept saturated until $H=-H_{R 1}$ is reached. Following the magnetization drop at $-H_{R 1}$ the total magnetization reduces and a smooth decrease of the two rare-earth XMCD signals is observed. Also during 
this stage, the Gd XMCD signal is always smaller and decreases faster than the $\mathrm{Tb}$ one. While plotting the difference between the $\overline{\mathrm{XMCD}}$ signals coming from $\mathrm{Gd}$ and $\mathrm{Tb}$ [ $\Delta(\mathrm{XMCD})]$ versus field $H$ (Fig. 6), at low field, the linear field dependence of $\Delta(\mathrm{XMCD})$ attributed to the interface DW compression is very similar to the one observed at 100 K (Fig. 4). Only a minor difference in the line slope may be noticed. Moreover, the minor loop achieved by sweeping the field from $+1 \mathrm{kOe}$ to -100 Oe then back to $1 \mathrm{kOe}$ at $30 \mathrm{~K}$ (not shown in the paper) is similar to the one obtained at 100 K.

However, the behavior of the sample at $30 \mathrm{~K}$ for lower field values differs significantly. The total magnetization and the two XMCD signals keep on decreasing until saturation is reached (no second magnetization reversal is observed). Also for $-100 \mathrm{Oe}>H>-200 \mathrm{Oe} \Delta(\mathrm{XMCD})$ field dependence is no longer linear and is even decreasing for field smaller than -200 Oe (Fig. 6). This implies that as the field increases, the angle between the $\mathrm{GdFe}$ and the $\mathrm{TbFe}$ average magnetization at the interface decreases. This is not consistent with the compression effect as discussed above and it suggests that at least in some part of the sample the angle between the two magnetizations at the interface decreases. This clearly means that the system cannot be considered as a unique lateral magnetic domain with a Bloch domain wall. The only mechanism to explain this behavior is to consider that some parts ofthe interface magnetization are saturated. Indeed, if the interface DW vanishes, $\mathrm{Tb}$ and $\mathrm{Gd}$ moments become parallel to the applied field and $\Delta(\mathrm{XMCD})$ becomes zero. In other words, for field larger than 200 Oe we assume the presence of lateral domains [Fig. 7(d)]. Some domains are totally saturated along the negative field direction and in others a DW is present at the interface. We can thus defined two possible domain states: the DW state and the $\mathrm{Sat}^{-}$state. The number of $\mathrm{Sat}^{-}$domains increases as the field is reduced and finally for $H=-1000$ Oe the sample is totally saturated as proved by magnetization and XMCD results

This magnetic configuration also allows us to explain the special minor loops observed at $30 \mathrm{~K}$. Figure 5 shows a minor loop obtained using XMCD and SQUID, for which the field is swept back around $H \approx-400$ Oe. Indeed, from the previous discussion, for $H \approx-400 \mathrm{Oe}$, part of the domains are in the saturated state and the other part are in the DW state [Fig. 7(e)]. As the field is increased from $H \approx$ $-400 \mathrm{Oe}$, the fact that the signal from Gd increases more than the signal from $\mathrm{Tb}$ is consistent with a DW decompression. The decompression is also evidenced by the slow magnetization increase observed by SQUID measurements and confirmed by the $\triangle$ (XMCD) linear field dependence (Fig. 6). The slope is, however, much lower since the decompression concerns only the domain remaining in the DW state. The slow increase of the XMCD Tb signal is coherent with the pinning of the DW by the TbFe layer as discussed for the $100 \mathrm{~K}$ measurements. For a small positive field close to $H_{R 1}$, the major part of the GdFe magnetization reverses, inducing a steep jump in the SQUID measurement. Compared to the previous $\mathrm{GdFe}$ magnetization reversal which occurred for $H=-H_{R 1}$, the effect on XMCD measurements is much larger. Indeed at $H=-H_{R 1}$ no significant XMCD variation

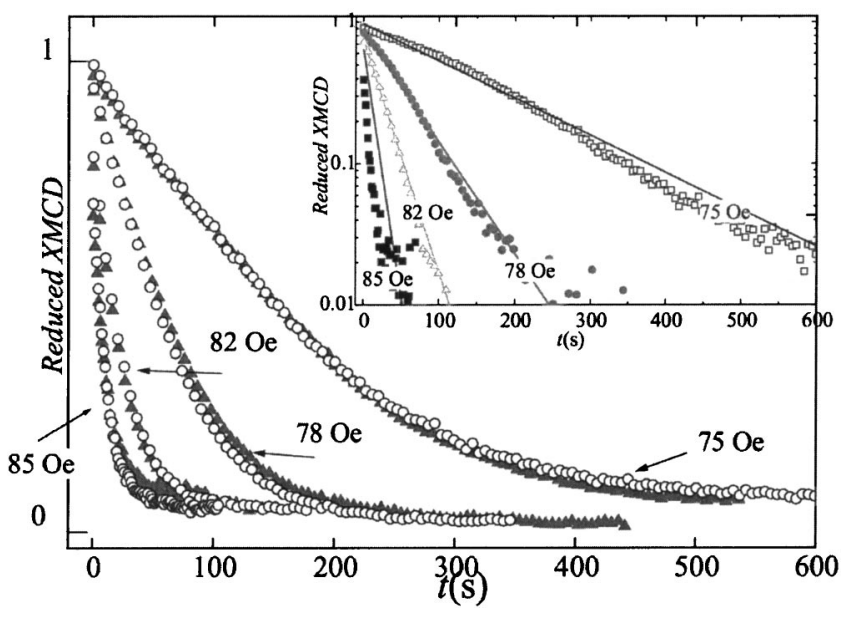

FIG. 8. Reduced XMCD signals at the Gd (solid symbol) and Tb (open symbol) $M_{4,5}$ edges collected as a function of time at $100 \mathrm{~K}$ for different applied fields. In the inset, the evolution of the reduced XMCD signal with time plotted in a logarithmic scale. The reduced XMCD signals are fitted by $\exp [-t / \tau(H)]$. For a given field each curve is characterized by a single relaxation time $[\tau(H)]$.

was observed whereas for $H=H_{R 1}$ it results in a jump of both Gd and Tb XMCD signals. This behavior may be understood considering the following scenario: The GdFe magnetization reversal switches all domain states [Figs. 7(e)7(f)]: the domains being in compressed DW states become saturated in the positive direction and the saturated domain switch to a DW state. As a domain in the $\mathrm{Sat}^{-}$state switched to a DW state, a small increase of the two XMCD signals is expected since the $\mathrm{GdFe} / \mathrm{TbFe}$ interface is weakly affected by such magnetization reversal. However, as a domain in the compressed DW states switches to the $\mathrm{Sat}^{+}$state, the compressed DW at the interface vanishes which lead to a sharp increase of both $\mathrm{Gd}$ and $\mathrm{Tb}$ signal. The slow increase of $\mathrm{Gd}$ and Tb signal following this jump is due to the DW compression and to domains switching from the DW state to the $\mathrm{Sat}^{+}$state.

Measurements performed at $30 \mathrm{~K}$ then show that two combined mechanisms explain the magnetization behavior of the $\mathrm{GdFe} / \mathrm{TbFe}$ interface: (i) the domain wall compression characterized by the difference between the orientation of the two alloy magnetization at the interface; (ii) the $\mathrm{GdFe} / \mathrm{TbFe}$ magnetization reversal resulting in the DW annihilation and the alignment of both alloy magnetization along the field. This reversal takes place on a large field range and the formation of lateral domains has to be taken into account. This behavior was not observed at $100 \mathrm{~K}$ for which a rather sharp $\mathrm{GdFe} / \mathrm{TbFe}$ magnetization reversal is observed.

\section{B. Aftereffect measurements}

In order to study the second magnetization reversal occurring for $H=-H_{R 2}$ at $T=100 \mathrm{~K}, \mathrm{XMCD}$ signal versus time experiments were carried out to get an aftereffect elementselective measurement. The sample was first saturated along 


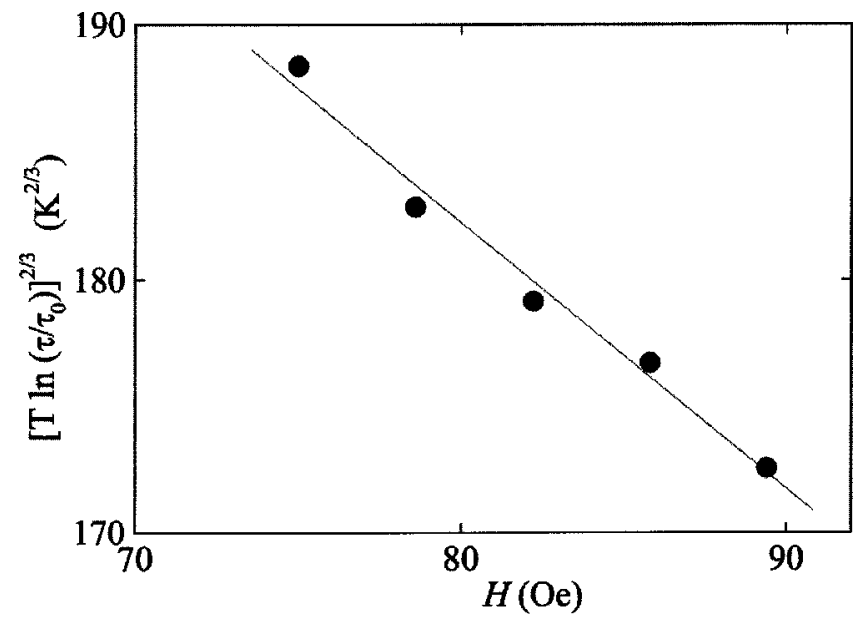

FIG. 9. $\left[T \ln \left(\tau / \tau_{0}\right)\right]^{2 / 3}$ as a function of the applied field $H$ where $\tau$ is the relaxation time deduced from relaxation measurements (Fig. $8)$ and $\tau_{0}$ is arbitrarily chosen $\left(\tau_{0}=10^{-10} \mathrm{~s}\right)$.

the positive field direction and the field was thus decreased to a value close to $-H_{R 2}$ (the time at which this value is reach is defined as $t=0$ ). The XMCD signals from the two rare earths were measured consecutively as a function of time as presented in Fig. 8. By this means the complete reversal of both alloy magnetization is monitored. In order to obtain the "reduced XMCD signal" shown in Fig. 8, the maximum XMCD signal at $t=0$ was normalized to 1 and its minimum, corresponding to the sample saturated along the negative field direction, to zero. $\mathrm{Gd}$ and $\mathrm{Tb}$ reduced signals are totally superimposed for all fields and present an exponential decay variation as shown in the inset of Fig. 8. The reduced XMCD signal could then be fitted by $\exp (-t / \tau)$ with a single relaxation time $\tau$ for each field value. The XMCD signal is found to decrease faster for larger field value.

Throughout this interface magnetization reversal, the sample switches from a state where a DW is present at the interface (DW state) [Fig. 7(c)] to a state for which TbFe magnetization is reversed (Sat state) [Fig. 7(g)]. The XMCD signal versus time results allow us to state that many events are taking place during this reversal process at $100 \mathrm{~K}$. This is definitively evidenced by the fact that the magnetization relaxation showed a continuous decrease of the magnetization with time. In the case of a single event process, a unique jump is expected. The simplest many events model is the one for which the bilayer is divided in lateral domains. Each domain can then be considered like a "domain wall junction" as in the model proposed by Gunther and Barbara. ${ }^{37}$ For fields larger than $-H_{R 2}$ all domains are in the DW state [Fig. 7(c)] and then a domain has a probability $P$ to have switched into the saturated state [Fig. $7(\mathrm{~d})]$ where $P$ as a function of time $(t)$ is given by

$$
P=1-\exp \left(-\frac{t}{\tau}\right)
$$

where the relaxation time $\tau$ is given by

$$
\tau=\tau_{0} \exp \left(\frac{\Delta E(H)}{k T}\right)
$$

with

$$
\Delta E(H)=\Delta E_{R 2}^{0}\left(1-\frac{H}{H_{R 2}^{0}}\right)^{3 / 2},
$$

where $\Delta E(H)$ is the energy barrier height ${ }^{37}$ to drop from one state to the other, with $\Delta E_{R 2}^{0}$ the energy barrier in zero field, $H_{R 2}^{0}$ the field for which the energy barrier vanishes, and $\Gamma_{0}$ $=1 / \tau_{0}$ the attempt frequency. Considering that each domain has identical size and same probability $P(t)$ to drop from one state to the other implies an exponential decay of the magnetization with respect to time, and a linear decrease of $\left[T \ln \left(\tau / \tau_{0}\right)\right]^{2 / 3}$ with respect to $H$ is expected [where $\Gamma_{0}$ $=1 / \tau_{0}$ is usually assume to range from $10^{6}$ to $10^{12} \mathrm{~Hz}$ (Ref. 38 ), in our case we have arbitrarily chosen $\tau_{0}=10^{-10} \mathrm{~s}$ ]. Figures 8 and 9 show that these effects are experimentally observed. Using this model, $\Delta E_{R 2}^{0}$ and $H_{R 2}^{0}$ are determined by extrapolation to the axis of the curve $\left[T \ln \left(\tau / \tau_{0}\right)\right]^{2 / 3}$ versus $H$ (Fig. 9). The estimated values are $\Delta E_{R 2}^{0} / k \approx 4300 \mathrm{~K}$ and $H_{R 2}^{0} \approx 250$ Oe which are comparable to values obtained by conventional aftereffect measurements on a similar system. ${ }^{7}$

Aftereffect measurements then permit us to unify the $30 \mathrm{~K}$ and $100 \mathrm{~K}$ results. Indeed for both temperatures lateral domain formation has to be assumed to explain the interface magnetization reversal. However, the difference between the two temperatures appears since at $100 \mathrm{~K}$ we observed a sharp interface magnetization reversal over a small field range and large aftereffect, whereas at $30 \mathrm{~K}$, no variation of XMCD signal versus time signals were detected for any applied field. This proves that for $30 \mathrm{~K}$ thermal activation is weaker, however, this is not sufficient to explain the overall behavior. Indeed the above model suggested that at $30 \mathrm{~K}$ the interface magnetization reversal would be observed for a field $H_{R 2}=100 \mathrm{Oe}<H<H_{R 2}^{0}=250 \mathrm{Oe}$. It then suggests that for a more complete description, the energy barrier distribution, the temperature dependence of the magnetic parameters, and the interaction between neighbor lateral domains should be taken into account. Also we restricted our analysis to a single type of magnetization reversal which may be defined as lateral domain nucleation whereas lateral domain growing (by domain wall propagation) should also be considered.

\section{CONCLUSIONS}

In this paper we have shown that the combination of regular SQUID measurements and element-specific and interfacesensitive XMCD measurements allow us to provide important information on the field, temperature, and time dependence of magnetization processes occurring in an exchange-coupled system. The magnetization reversal process in a spring magnet $\mathrm{GdFe} / \mathrm{TbFe}$ has been studied using both quasistatic and dynamic magnetization measurements. First of all, XMCD measurements provided the value of the magnetic moment held by the species involved at the interface in the two GdFe and TbFe ferrimagnetic alloys. Then 
joint XMCD and SQUID results allowed us to confirm that as the magnetization of the soft layer reverses, an interface DW mostly located in the soft layer is created. Evidence of the compression of this domain wall and its pinning at the interface of the soft and hard layers was also obtained. The interface magnetization reversal was shown to take place by lateral domain switching. For $100 \mathrm{~K}$ this reversal was observed in a narrow field range. Aftereffect measurements permitted us to study this thermally activated magnetization reversal. However, for $30 \mathrm{~K}$ both domain wall compression and lateral domain switching were observed on a large field range. This points out that for a full understanding of magnetization reversal in exchange coupled bilayer system both interface DW (as suggested by Mauri et al. ${ }^{16}$ ) and lateral domain formation ${ }^{15}$ have to be taken into account.

\section{ACKNOWLEDGMENTS}

The authors thank S. Pizzini and J. Vogel for help with the experiments and for fruitful and pleasant discussions.
${ }^{1}$ A. E. Berkowitz and K. Takano, J. Magn. Magn. Mater. 200, 552 (1999).

${ }^{2}$ J. Nogues and I. K. Schuller, J. Magn. Magn. Mater. 192, 203 (1999)

${ }^{3}$ Miguel Kiwi, J. Magn. Magn. Mater. 234, 584 (2001).

${ }^{4}$ J. Nogues, D. Lederman, T. J. Moran, and Ivan K. Schuller, Phys. Rev. Lett. 76, 4624 (1996).

${ }^{5}$ E. E. Fullerton, J. S. Jiang, M. Grimsditch, C. H. Sowers, and S. D. Bader, Phys. Rev. B 58, 12193 (1998).

${ }^{6}$ Shi-Shen Yan, J. A. Barnard, Feng-ting Xu, J. L. Weston, and G. Zangari, Phys. Rev. B 64, 184403 (2001).

${ }^{7}$ S. Mangin, G. Marchal, and B. Barbara, Phys. Rev. Lett. 82, 4336 (1999); S. Mangin, A. Sulpice, G. Marchal, C. Bellouard, W. Wernsdorfer, and B. Barbara, Phys. Rev. B 60, 1204 (1999).

${ }^{8}$ R. Rohlsberger, H. Thomas, K. Schlage, E. Burkel, O. Leupold, and R. Ruffer, Phys. Rev. Lett. 89, 237201 (2002).

${ }^{9}$ V. E. Kuncser, M. Doi, W. Keune, M. Askin, H. Spies, J. S. Jiang, A. Inomata, and S. D. Bader, Phys. Rev. B 68, 064416 (2003).

${ }^{10}$ C. Tiusan, T. Dimopoulos, K. Ounadjela, M. Hehn, H. A. M. van den Berg, V. da Costa, and Y. Henry, Phys. Rev. B 61, 580 (2000).

${ }^{11}$ F. Canet, S. Mangin, C. Bellouard, and M. Piecuch, Europhys. Lett. 52, 594 (2000).

${ }^{12}$ S. Wuchner, J. C. Toussaint, and J. Voiron, Phys. Rev. B 55, 11576 (1997).

${ }^{13}$ J. R. Childress et al., IEEE Trans. Magn. 37, 1745 (2001).

${ }^{14}$ B. Dieny, V. S. Speriosu, S. S. P. Parkin, J. C. Scott, B. A. Gurney, D. R. Wilhoit, and D. Mauri, Phys. Rev. B 43, 1297 (1991).

${ }^{15}$ A. P. Malozemoff, J. Appl.Phys. 63, 3874 (1988).

${ }^{16}$ D. Mauri, H. C. Siegmann, P. S. Bagus, and E. Kay, J. Appl. Phys. 62, 3047 (1987).

${ }^{17}$ R. L. Stamps, J. Magn. Magn. Mater. 242-245, 139 (2002).

${ }^{18}$ M. Kiwi, J. Mejia-lopez, R. D. Portugal, and R. Ramirez, Europhys. Lett. 48, 573 (1999).

${ }^{19}$ F. Montaigne, S. Mangin, and Y. Henry, Phys. Rev. B 67, 144412 (2003).

${ }^{20}$ V. Chakarian, Y. U. Idzerda, G. Meigs, E. E. Chaban, J. H. Park, and C. T. Chen, Appl. Phys. Lett. 66, 3368 (1995).

${ }^{21}$ J. W. Freeland, V. Chakarian, Y. U. Idzerda, S. Doherty, J. G. Zhu, H. Wende, and C. C. Kao, J. Vac. Sci. Technol. A 16, 1355
(1998).

${ }^{22}$ K. Dumesnil, C. Dufour, Ph. Mangin, and A. Rogalev, Phys. Rev. B 65, 094401 (2002).

${ }^{23}$ M. Bonfim, G. Ghiringhelli, F. Montaigne, S. Pizzini, N. B. Brookes, F. Petroff, J. Vogel, J. Camarero, and A. Fontaine, Phys. Rev. Lett. 86, 3646 (2001).

${ }^{24}$ T. Yonamine, A. P. B. Tufaile, J. Vogel, A. D. Santos, F. C. Vicentin, and H. C. N. Tolentino, J. Magn. Magn. Mater. 233, 84 (2001).

${ }^{25}$ H. Ohldag, T. J. Regan, J. Stöhr, A. Scholl, F. Nolting, J. Lüning, C. Stamm, S. Anders, and R. L. White, Phys. Rev. Lett. 87, 247201 (2001).

${ }^{26}$ H. Ohldag, A. Scholl, F. Nolting, S. Maat, A. T. Young, M. Carey, and J. Stöhr, Phys. Rev. Lett. 91, 017203 (2003).

${ }^{27}$ C. Brouder and J. P. Kappler, in Magnetism and Synchrotron Radiation, edited by E. Beaurepaire, B. Carrière, and J. P. Kappler (Les éditions de Physique, Les Ulis, 1997).

${ }^{28}$ R. Nakajima, J. Stöhr, and Y. U. Idzerda, Phys. Rev. B 59, 6421 (1999).

${ }^{29}$ P. Hansen, C. Clausen, G. Much, M. Rosenkranz, and K. Witter, J. Appl. Phys. 66, 756 (1989).

${ }^{30}$ S. Mangin, C. Bellouard, G. Marchal, and B. Barbara, J. Magn. Magn. Mater. 165, 161 (1997).

${ }^{31}$ P. Cara, B. T. Thole, M. Altarelli, and X. Wang, Phys. Rev. Lett. 70, 694 (1993).

${ }^{32}$ C. T. Chen, Y. U. Idzerda, H. J. Lin, N. V. Smith, G. Meigs, E. Chaban, G. H. Ho, E. Pellegrin, and F. Sette, Phys. Rev. Lett. 75, 152 (1995).

${ }^{33}$ M. Altarelli and P. Sainctavit, in Magnetism and Synchrotron Radiation, edited by E. Beaurepaire, B. Carriere, and J. P. Kappler (Les éditions de Physique, Les Ulis, 1997).

${ }^{34}$ K. Moorjani and J. M. D. Coey, Magnetic Glasses (Elsevier Science, New York, 1984).

${ }^{35}$ R. W. Cochrane, R. Harris, and M. J. Zuckermann, Phys. Rep. 48, 1 (1978).

${ }^{36}$ S. Mangin, F. Montaigne, C. Bellouard, H. Fritzsche, Appl. Phys. A: Mater. Sci. Process. XX, S631 (2002).

${ }^{37}$ L. Gunther and B. Barbara, Phys. Rev. B 49, 3926 (1994).

${ }^{38}$ M. Labrune, S. Andrieu, F. Rio, and P. Bernstein, J. Magn. Magn. Mater. 80, 211 (1989). 